\title{
Treatment of Plantar Ulcers with Split Skin Grafts: A Simplified Method
}

\author{
P. J. D'HOOGHE AND E. HENDRICKX \\ All Africa Leprosy and Rehabilitation Training Centre (ALERT) \\ Department of Surgery, Addis Abaha, Ethiopia
}

\begin{abstract}
The advantages of skin grafting versus plaster of Paris in the treatment of plantar ulcers are briefly discussed. A simplified method of split thickness skin grafting (prelevement, anaesthesia and storage of skin graft) is presented.
\end{abstract}

Split skin grafting of plantar ulcers is a method of treatment long favoured by plastic surgeons, its indications of ten being considered the same as those for plaster of Paris treatment, i.e. the superficial, uncomplicated ulcer. Plaster of Paris treatment has the advantage that it enables the patient to be ambulatory. It has the disadvantage that it prevents control of the wound and the early detection of complications. Immobilization with plaster of Paris is also liable to enhance osteoporosis, with the risk of fracture, going on to neuropathic disintegration of the foot.

The epidermis is a simple tissue. Following "the erosion" of its loss, it can be reconstituted ad integrum by regeneration of the epithelium. The dermis however, is a complex organ, and in mammals such organs do not replace themselves; they heal with the formation of scar tissue. An ulcer is a defect of the total thickness of skin, dermis and epidermis. Healing takes place by the formation of scar tissue (contracting collagenous fibres) combined with epithelization. This combination, scar and epithelium, is an inadequate substitute for skin, which, especially in a weight bearing area, must be strong and elastic. The skin grafting of ulcers therefore must be preferred, whenever possible, to the healing "per secundam intentionem", obtained with the conservative plaster of Paris method. Of course, the most beautiful skin graft does not resolve the basic problem of denervation and insensitivity, and all methods of preventing the recurrence of ulceration (avoiding weightbearing, wearing of protective shoes, etc.) must be scrupulously continued.

It has been our aim to simplify the skin grafting procedure as much as possible, and describe here some technical details which have given constant satisfaction over a long period of time.

\section{Choice of Donor Area and Anaesthesia}

Some surgeons take the skin graft in patients with leprosy from an insensitive area of the lower leg, thus dispensing with the need for any anaesthetic. Denervated dermis however is usually thinner than normal dermis, and the Received for publication 22nd November, 1974. 
adnexae, hair follicles and skin glands which contribute much to the healing and re-epithelization of the donor area may also be atrophic, so that delay in healing of the donor area. and sometimes full thickness skin loss are not negligible risks of this procedure. On the other hand, the lateral aspect of the thigh, often not affected by the disease, is a very suitable donor area for skin grafts.

The lateral femoral cutaneous nerve provides sensation to this area, limited medially by a line which can be drawn from the anterior iliac spine to the lateral side of the patella. Regional block by infiltration of this nerve is easy to perform.

The nerve perforates the lateral part of the inguinal ligament about $1 \mathrm{~cm}$ medial to and below the anterior iliac spine. The anterior iliac spine is located by palpation, and a fine $5 \mathrm{~cm}$ needle is introduced perpendicularly to the skin, until its point comes in contact with the margin of the iliac bone, from which point $5 \mathrm{ml}$ of $1 \%$ xylocaine solution are injected in a "fanwise" manner, parallel to Poupart's ligament and up to approximately $5 \mathrm{~cm}$ from it.

\section{Instrumentarium}

When one of the classic and rather expensive dermatomes is not available, surgeons often resort to the old Reverdin method of "pinch grafting", or use the "free hand" technique. The latter method is difficult, and even skilled hands often obtain only poor grafts, of irregular thickness and unpredictable dimensions. The former method cannot be recommended, as it leaves unsightly scars on the donor area.

Beautiful grafts, of perfectly constant and useful split thickness type (12/1000 of an inch) can be cut with a Schick injector safety razor. The technique is simple, and is illustrated in the accompanying photograph. After lubricating skin and razor, the apparatus is pressed firmly against the skin, with the same inclination as

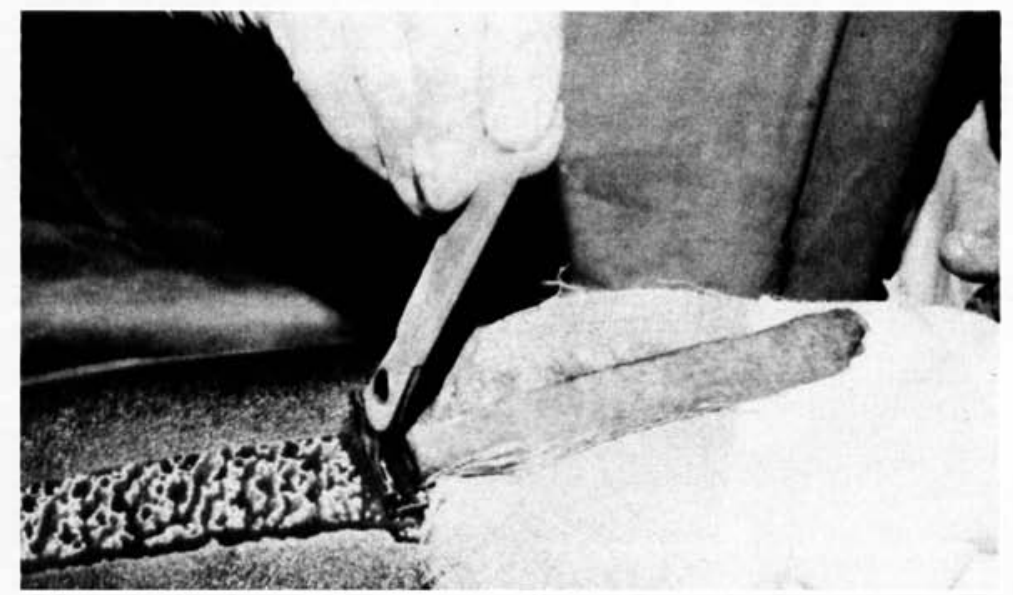

Fig. 1. Technique of prelevating the split skin graft with a Schick safety razor.

used in shaving, and pushed forwards without any movement transversely. The prelevated skin graft is about $3 \mathrm{~cm}$ wide, and is limited in length only by the limits of anaesthesia. Multiple strips can be prelevated side by side, as rapidly as with an electric dermatome. The handle of the commercial razor not being heat 
resistant, the instrument must be adapted for sterilization, and the handle replaced by a thermoresistant one.

\section{Storage of Surplus Skin Graft}

Sometimes it can be useful if a surplus graft can be taken and stored for use in case of failure. A number of methods have been advocated for preserving autogenous skin grafts. The one enjoying most widespread use, and in theory permitting the graft to be used up to three weeks after prelevement, is refrigeration in saline at a temperature between $2^{\circ} \mathrm{C}$ and $6^{\circ} \mathrm{C}$. A refrigerator, however is not always available, and experience with grafts preserved following this method is often disappointing. It is not difficult to understand why test-tube preserved grafts seldom "take", because even the most carefully prepared skin contains living bacteria hidden in the adnexae in almost every case. The preserved skin, which regularly yields positive cultures of staphylococcus and maybe pseudomonas, is no longer protected by the body, and is just a culture medium for micro-organisms.

If the surplus graft is re-applied and stored on the donor bed, care being taken to dry the donor bed prior to replacement of the graft, the graft will soon have a demonstrable capillary connection with the remaining dermis, and enjoy the protection of the body's defence mechanisms.

Either the first transplantation will succeed; in that eventuality the stored graft can be left, and the donor scar is limited to the minimum. In the other eventuality, a supplementary graft is needed. The stored graft can gently be removed by forceps traction for up to 14 days. The transfer can be done during a dressing change, at the bedside, without the need for an anaesthetic, and the "take" is as satisfying as with a freshly prelevated graft.

\section{Acknowledgement}

We wish to acknowledge our indebtedness to the Damien Foundation, Belgium, for their support and encouragement. 